\title{
Nucleophilic Attack at Selenium in Diselenides and Selenosulfides.
}

\section{A Computational Study.}

Steven M. Bachrach, ${ }^{*}$ Dustin W. Demoin, Michelle Luk and James V. Miller Jr.

Department of Chemistry

Trinity University

1 Trinity Place,

San Antonio, TX 78212

\section{Supporting Information Available}

Coordinates of all optimized structures, their absolute energies, and number of imaginary

frequencies.

Table of Contents

Reaction $1 \ldots \ldots . . .2$

Reaction $2 \ldots \ldots . . .3$

Reaction 3........ 4

Reaction 4........6

Reaction 5........7

Reaction 6........7 7

Reaction $7 \ldots \ldots \ldots .8$

Reaction $8 \ldots \ldots \ldots .8$

Reaction 9........ 9

Reaction $10 \ldots . . .10$ 


$$
\begin{array}{lrrr}
\text { 1s-TS at B3LYP/6-31+G* } & \\
\mathrm{Se} & -0.371047 & 0.652713 & 0.013416 \\
\mathrm{Se} & -2.564849 & -0.366716 & -0.050257 \\
\mathrm{H} & 0.615580 & -0.503927 & -0.082044 \\
\mathrm{H} & -2.733182 & -0.604662 & 1.412267 \\
\mathrm{Se} & 2.914038 & -0.243561 & -0.044665 \\
\mathrm{H} & 2.860767 & -0.334237 & 1.440988 \\
\mathrm{E}= & -7200.0596461 & \mathrm{NIMAG}=1
\end{array}
$$

\section{1s-TS at MP2/6-31+G*}

$$
\begin{array}{lrrr}
\mathrm{Se} & -0.396130 & 0.655607 & 0.014168 \\
\mathrm{Se} & -2.543774 & -0.371861 & -0.049912 \\
\mathrm{H} & 0.516132 & -0.529305 & -0.104996 \\
\mathrm{H} & -2.685326 & -0.632503 & 1.405628 \\
\mathrm{Se} & 2.918841 & -0.239786 & -0.044806 \\
\mathrm{H} & 2.885335 & -0.332863 & 1.438060 \\
\mathrm{E}= & -7194.9128761 \mathrm{NIMAG}=1
\end{array}
$$

\section{1s-TS at B3LYP/aug-cc-pVDZ}

$$
\begin{array}{lrrr}
\mathrm{Se} & -0.366735 & 0.651401 & 0.009418 \\
\mathrm{Se} & -2.597547 & -0.366561 & -0.047906 \\
\mathrm{H} & 0.643929 & -0.487896 & -0.042466 \\
\mathrm{H} & -2.763362 & -0.544091 & 1.416808 \\
\mathrm{Se} & 2.939698 & -0.245792 & -0.044226 \\
\mathrm{H} & 2.955284 & -0.295629 & 1.437910 \\
\mathrm{E}= & -7206.5734526 \mathrm{NIMAG}=1
\end{array}
$$

\begin{tabular}{|c|c|c|}
\hline \multicolumn{3}{|c|}{ s-INT at MP2/aug-cc-pVDZ } \\
\hline-0.084208 & 1.326740 & 0.000000 \\
\hline 0.02 & -0.131154 & 000 \\
\hline 892 & 0.048674 & 941 \\
\hline 892 & 0.048674 & -2.689415 \\
\hline 86 & 8681 & 2.784947 \\
\hline-1 & 81 & 3947 \\
\hline
\end{tabular}

\section{1s-TS at MP2/aug-cc-pVDZ}

$\begin{array}{llll}\mathrm{Se} & -0.376910 & 0.649237 & 0.012453\end{array}$

$\mathrm{Se}-2.555114-0.366455-0.049180$

$\mathrm{H} \quad 0.545501-0.520376-0.073861$

$\mathrm{H} \quad-2.690875-0.575590 \quad 1.400712$

Se $2.911074-0.240608-0.044167$

$\mathrm{H} \quad 2.857645 \quad-0.337995 \quad 1.423564$

$\mathrm{E}=-7201.6979073 \mathrm{NIMAG}=1$

\section{1s-INT at B3LYP/6-31+G* \\ $\mathrm{H} \quad-0.338886 \quad 1.353675 \quad 0.000000$ \\ Se $\quad 0.030768-0.077081 \quad 0.000000$ \\ $\begin{array}{llll}\text { Se } & 0.030768 & 0.028947 & 2.679298\end{array}$ \\ Se $\quad 0.030768 \quad 0.028947-2.679298$ \\ $\mathrm{H} \quad-1.399734-0.350654 \quad 2.840429$ \\ $\mathrm{H} \quad-1.399734-0.350654-2.840429$ \\ $\mathrm{E}=-7200.0756825 \mathrm{NIMAG}=0$}

\section{1s-INT at MP2/6-31+G*}

$\begin{array}{lrrr}\mathrm{H} & -0.083282 & 1.374186 & 0.000000 \\ \mathrm{Se} & 0.029101 & -0.096518 & 0.000000 \\ \mathrm{Se} & 0.029101 & 0.031188 & 2.647473 \\ \mathrm{Se} & 0.029101 & 0.031188 & -2.647473 \\ \mathrm{H} & -1.442511 & -0.106671 & 2.779759 \\ \mathrm{H} & -1.442511 & -0.106671 & -2.779759 \\ \mathrm{E}= & -7194.9289269 & \mathrm{NIMAG}=0\end{array}$

\section{1s-INT at B3LYP/aug-cC-pVDZ}

$\begin{array}{lrrr}\mathrm{H} & -0.336032 & 1.329732 & 0.000000 \\ \mathrm{Se} & 0.030659 & -0.097160 & 0.000000 \\ \mathrm{Se} & 0.030659 & 0.038939 & 2.728097 \\ \mathrm{Se} & 0.030659 & 0.038939 & -2.728097 \\ \mathrm{H} & -1.395604 & -0.337080 & 2.877371 \\ \mathrm{H} & -1.395604 & -0.337080 & -2.877371 \\ \mathrm{E}= & -7206.5881242 \mathrm{NIMAG}=0\end{array}$

\section{1a-TS at B3LYP/6-31+G*}

$\begin{array}{lrrr}\text { Se } & -0.369786 & 0.648852 & -0.003790 \\ \mathrm{Se} & -2.565886 & -0.367394 & 0.046038 \\ \mathrm{H} & 0.617289 & -0.510708 & -0.038420 \\ \mathrm{H} & -2.777612 & -0.494398 & -1.424530 \\ \mathrm{Se} & 2.914341 & -0.243539 & -0.041775 \\ \mathrm{H} & 2.885581 & -0.284139 & 1.446872 \\ \mathrm{E}= & -7200.0596423 \mathrm{NIMAG}=0\end{array}$

$$
\begin{aligned}
& \text { 1a-TS at MP2/6-31+G* } \\
& \begin{array}{lrrr}
\mathrm{Se} & -0.395841 & 0.653623 & -0.005653 \\
\mathrm{Se} & -2.543624 & -0.374059 & 0.046989 \\
\mathrm{H} & 0.515614 & -0.537796 & -0.020250 \\
\mathrm{H} & -2.735912 & -0.512718 & -1.419439 \\
\mathrm{Se} & 2.918626 & -0.241420 & -0.041479 \\
\mathrm{H} & 2.928806 & -0.246381 & 1.444553 \\
\mathrm{E}= & -7194.9128828 & \text { NIMAG }=0
\end{array}
\end{aligned}
$$

$$
\begin{array}{lrrr}
\text { 1a-TS at B3LYP/aug-cc-pVDz } \\
\text { Se }-0.366522 & 0.650398 & -0.004441 \\
\mathrm{Se} & -2.597516 & -0.367270 & 0.046223 \\
\mathrm{H} & 0.644110 & -0.489872 & -0.016063 \\
\mathrm{H} & -2.781618 & -0.498178 & -1.421265 \\
\mathrm{Se} & 2.939738 & -0.246086 & -0.041882 \\
\mathrm{H} & 2.963726 & -0.271341 & 1.440742 \\
\mathrm{E}= & -7206.5734471 & \mathrm{NIMAG}=0
\end{array}
$$

\section{1a-TS at MP2/aug-cc-pVDZ}

$\begin{array}{lrrr}\text { Se } & -0.377001 & 0.648852 & -0.003448 \\ \text { Se } & -2.554626 & -0.368864 & 0.044915 \\ \mathrm{H} & 0.545485 & -0.523760 & -0.020197 \\ \mathrm{H} & -2.724840 & -0.486639 & -1.411646 \\ \mathrm{Se} & 2.910982 & -0.242063 & -0.041404 \\ \mathrm{H} & 2.881302 & -0.279046 & 1.429692 \\ \mathrm{E}= & -7201.6978918 \mathrm{NIMAG}=0\end{array}$




\section{1a-INT at B3LYP/6-31+G* \\ $\mathrm{H} \quad 0.000000 \quad 0.000000-1.394530$ \\ $\begin{array}{lllll}\text { Se } & 0.000000 & 0.000000 & 0.083977\end{array}$ \\ $\begin{array}{llll}\text { Se } & 0.000000 & 2.679490 & -0.020856\end{array}$ \\ Se $\quad 0.000000-2.679490-0.020856$ \\ $\mathrm{H} \quad-1.481359 \quad 2.826999-0.021217$ \\ $\mathrm{H} \quad 1.481359-2.826999-0.021217$ \\ $\mathrm{E}=-7200.0756148 \mathrm{NIIMAG}=0$}

\section{1a-INT at MP2/6-31+G*}

$\mathrm{H} \quad 0.000000 \quad 0.000000 \quad-1.376701$

$\begin{array}{llll}\text { Se } & 0.000000 & 0.000000 & 0.098180\end{array}$

Se $0.000000 \quad 2.647911-0.028803$

Se $\quad 0.000000-2.647911-0.028803$

$\mathrm{H} \quad-1.478287 \quad 2.774742 \quad-0.001414$

$\mathrm{H} \quad 1.478287 \quad-2.774742 \quad-0.001414$

$\mathrm{E}=-7194.928869 \mathrm{NIMAG}=0$

\section{1a-INT at B3LYP/aug-CC-pVDZ \\ H $\quad 0.000000 \quad 0.000000-1.374790$ \\ $\begin{array}{llll}\text { Se } & 0.000000 & 0.000000 & 0.099322\end{array}$ \\ $\begin{array}{llll}\text { Se } & 0.000000 & 2.728801 & -0.029577\end{array}$ \\ Se $\quad 0.000000-2.728801-0.029577$ \\ $\begin{array}{llll}\mathrm{H} & -1.476218 & 2.857328 & 0.004542\end{array}$ \\ $\mathrm{H} \quad 1.476218 \quad-2.857328 \quad 0.004542$ \\ $\mathrm{E}=-7206.5880875 \mathrm{NIMAG}=0$}

\section{1s-INT at MP2/aug-cc-pVDZ}

$\begin{array}{lrrr}\mathrm{H} & 0.000000 & 0.000000 & -1.329329 \\ \mathrm{Se} & 0.000000 & 0.000000 & 0.133010 \\ \mathrm{Se} & 0.000000 & 2.689722 & -0.046440 \\ \mathrm{Se} & 0.000000 & -2.689722 & -0.046440 \\ \mathrm{H} & -1.466901 & 2.777687 & -0.017559 \\ \mathrm{H} & 1.466901 & -2.777687 & -0.017559 \\ \mathrm{E}= & -7201.7126812 \mathrm{NIMAG}=0\end{array}$

\section{2s-TS at B3LYP/6-31+G*}

Se $0.313058-0.655130 \quad 0.451690$

Se $2.557197-0.148946-0.301489$

$\mathrm{H} \quad-0.610372-0.302947-0.702855$

Se $-2.875988-0.173974-0.247757$

C $\quad 2.602592 \quad 1.779409 \quad 0.125555$

$\mathrm{H} \quad 1.878550 \quad 2.316640 \quad-0.491919$

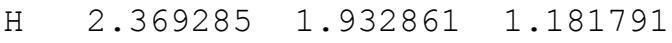

H $\quad 3.612598 \quad 2.147451-0.088092$

C $\quad-2.542524 \quad 1.712330 \quad 0.262810$

$\mathrm{H} \quad-3.021382 \quad 1.941928 \quad 1.221149$

$\mathrm{H} \quad-1.462759 \quad 1.871957 \quad 0.367047$

$\mathrm{H} \quad-2.931408 \quad 2.395373-0.500393$

$\mathrm{E}=7278.6949924 \mathrm{NIMAG}=1$

\section{2s-TS at MP2/6-31+G*}

Se $0.328790-0.714958 \quad 0.433503$

Se $2.510145-0.114582-0.300830$

$\mathrm{H} \quad-0.525637-0.355591-0.748382$

Se $-2.853075-0.140616-0.247827$

$\begin{array}{llll}\text { C } & 2.417520 & 1.791818 & 0.143777\end{array}$

$\mathrm{H} \quad 1.663208 \quad 2.280541-0.475956$

$\mathrm{H} \quad 2.160805 \quad 1.910149 \quad 1.198017$

H $\quad 3.400557 \quad 2.231409-0.053555$

C $\quad-2.337372 \quad 1.680996 \quad 0.311091$

$\mathrm{H} \quad-2.767647 \quad 1.915935 \quad 1.289501$

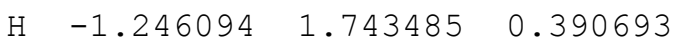

$\mathrm{H} \quad-2.685306 \quad 2.422484 \quad-0.414323$

$\mathrm{E}=-7273.2536936 \mathrm{NIMAG}=1$

$\begin{array}{lrrr}\text { 2s-INT at B3LYP/6-31+G* } \\ \text { H } & 1.386307 & -0.433217 & 0.000000 \\ \mathrm{Se} & -0.092068 & -0.404115 & 0.000000 \\ \mathrm{Se} & 0.020386 & -0.274904 & 2.665751 \\ \mathrm{Se} & 0.020386 & -0.274904 & -2.665751 \\ \mathrm{C} & 0.020386 & 1.701434 & 2.720229 \\ \mathrm{C} & 0.020386 & 1.701434 & -2.720229 \\ \mathrm{H} & 0.945682 & 2.097913 & 2.290455 \\ \mathrm{H} & 0.945682 & 2.097913 & -2.290455 \\ \mathrm{H} & -0.828233 & 2.094638 & -2.152283 \\ \mathrm{H} & -0.828233 & 2.094638 & 2.152283 \\ \mathrm{H} & -0.060890 & 2.032144 & 3.761795 \\ \mathrm{H} & -0.060890 & 2.032144 & -3.761795 \\ \mathrm{E}=-7278.7097133 & \mathrm{NIMAG} & =\end{array}$




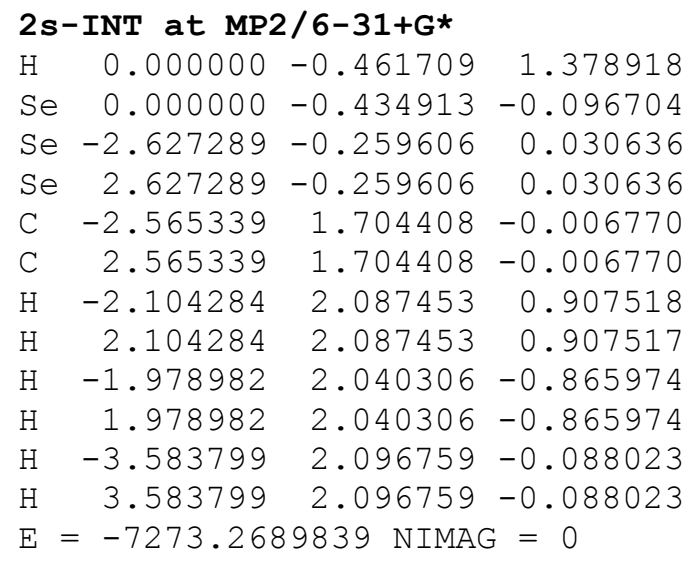

2a-TS at B3LYP/6-31+G*

$\begin{array}{lrrr}\text { Se } & -0.305698 & -0.005393 & 0.656387 \\ \mathrm{Se} & -2.507658 & 0.463558 & -0.236847 \\ \mathrm{H} & 0.580941 & -0.259117 & -0.550740 \\ \mathrm{Se} & 2.851116 & -0.480878 & -0.171193 \\ \mathrm{C} & -3.164508 & -1.385995 & -0.472509 \\ \mathrm{H} & -2.527601 & -1.917967 & -1.182958 \\ \mathrm{H} & -3.162974 & -1.909576 & 0.486400 \\ \mathrm{H} & -4.187322 & -1.333031 & -0.862864 \\ \mathrm{C} & 2.992913 & 1.486773 & -0.376558 \\ \mathrm{H} & 3.492123 & 1.742570 & -1.317599 \\ \mathrm{H} & 1.989304 & 1.926595 & -0.376442 \\ \mathrm{H} & 3.561252 & 1.918109 & 0.454794 \\ \mathrm{E}= & 7278.6945883 \mathrm{NIMAG}=1\end{array}$

2a-TS at MP2/6-31+G*

$\begin{array}{lrrr}\text { Se } & -0.313848 & -0.028202 & 0.662244 \\ \text { Se } & -2.461230 & 0.460692 & -0.238994 \\ \text { H } & 0.504104 & -0.282391 & -0.571955 \\ \text { Se } & 2.844590 & -0.461647 & -0.181483 \\ \text { C } & -3.093973 & -1.380124 & -0.479269 \\ \text { H } & -2.449440 & -1.899264 & -1.190611 \\ \text { H } & -3.081473 & -1.902140 & 0.479000 \\ \text { H } & -4.116706 & -1.337193 & -0.867547 \\ \text { C } & 2.819092 & 1.506853 & -0.345661 \\ \text { H } & 3.299780 & 1.823806 & -1.275885 \\ \text { H } & 1.783827 & 1.863851 & -0.340829 \\ \text { H } & 3.345793 & 1.964319 & 0.497301 \\ \text { E }= & -7273.2527429 \text { NIMAG }=1\end{array}$

\section{2a-INT at B3LYP/6-31+G*}

$\begin{array}{lrrr}\mathrm{H} & 0.000000 & 0.000000 & -1.403707 \\ \mathrm{Se} & 0.000000 & 0.000000 & 0.075236 \\ \mathrm{Se} & 0.000000 & 2.669327 & -0.029128 \\ \mathrm{Se} & 0.000000 & -2.669327 & -0.029128 \\ \mathrm{C} & -1.968169 & 2.853482 & 0.040925 \\ \mathrm{C} & 1.968169 & -2.853482 & 0.040925 \\ \mathrm{H} & -2.367342 & 2.301157 & 0.896779 \\ \mathrm{H} & 2.367342 & -2.301157 & 0.896779 \\ \mathrm{H} & -2.422873 & 2.462542 & -0.874511 \\ \mathrm{H} & 2.422873 & -2.462542 & -0.874511 \\ \mathrm{H} & -2.226647 & 3.913245 & 0.145361 \\ \mathrm{H} & 2.226647 & -3.913245 & 0.145361 \\ \mathrm{E}= & -7278.7092312 \mathrm{NIMAG}=0\end{array}$

\section{2a-INT at MP2/6-31+G*}

$\begin{array}{lrrr}\mathrm{H} & 0.000000 & 0.000000 & -1.406392 \\ \mathrm{Se} & 0.000000 & 0.000000 & 0.069787 \\ \mathrm{Se} & 0.000000 & 2.632790 & -0.044212 \\ \mathrm{Se} & 0.000000 & -2.632790 & -0.044212 \\ \mathrm{C} & -1.958232 & 2.737752 & 0.103420 \\ \mathrm{C} & 1.958232 & -2.737752 & 0.103420 \\ \mathrm{H} & -2.293557 & 2.160889 & 0.968802 \\ \mathrm{H} & 2.293557 & -2.160889 & 0.968802 \\ \mathrm{H} & -2.428539 & 2.332491 & -0.796225 \\ \mathrm{H} & 2.428539 & -2.332491 & -0.796225 \\ \mathrm{H} & -2.255894 & 3.783596 & 0.226937 \\ \mathrm{H} & 2.255894 & -3.783596 & 0.226937 \\ \mathrm{E}= & -7273.2682254 \text { NIMAG }=0\end{array}$

\section{3s-TS at B3LYP/6-31+G*}

$$
\begin{array}{lrrr}
\text { Se } & -1.187416 & 0.940344 & 0.008194 \\
\text { C } & 0.412833 & -0.193193 & -0.009908 \\
\text { Se } & -2.944908 & -0.637912 & -0.046843 \\
\text { H } & 1.310964 & 0.444114 & -0.026828 \\
\text { H } & 0.444762 & -0.808210 & 0.890042 \\
\text { H } & 0.417201 & -0.820097 & -0.901945 \\
\text { H } & -3.058279 & -0.882358 & 1.421121 \\
\text { Se } & 3.967559 & -0.200728 & -0.042897 \\
\mathrm{H} & 4.010361 & -0.232253 & 1.449631 \\
\mathrm{E}=-7239.3715658 & \text { NIMAG }=1
\end{array}
$$

\footnotetext{
3s-TS at MP2/6-31+G*

$\begin{array}{llll}\text { Se } & -1.134501 & 0.918751 & 0.012653\end{array}$ C $\quad 0.359288-0.313328-0.144155$

Se $-2.914768-0.594394-0.031115$

$\mathrm{H} \quad \begin{array}{llll}1.275030 & 0.286120 & -0.190471\end{array}$

$\mathrm{H} \quad 0.400186-0.961215 \quad 0.731330$

$\mathrm{H} \quad 0.257089-0.898300-1.058080$

$\mathrm{H} \quad-2.937899-0.912786 \quad 1.420482$

Se $\quad 3.861677-0.206795 \quad 0.020890$

$\mathrm{H} \quad \begin{array}{llll}5.227968 & 0.369051 & -0.120905\end{array}$

$E=-7234.081064$ NIMAG $=1$
} 


\section{3s-INT at B3LYP/6-31+G* \\ C $\quad-0.049788 \quad 1.842158 \quad-0.007974$ \\ Se $-0.001200-0.143406-0.027640$ \\ Se $-2.680610-0.188991-0.027925$ \\ Se $2.694565-0.178502-0.028155$ \\ $\mathrm{H} \quad-2.814679-0.131139 \quad 1.453287$ \\ $\mathrm{H} \quad 2.835951 \quad-0.120233 \quad 1.452518$ \\ $\mathrm{H} \quad-0.661585 \quad 2.197997 \quad-0.839213$ \\ $\mathrm{H} \quad-0.476821 \quad 2.189136 \quad 0.936375$ \\ $\mathrm{H} \quad 0.982205 \quad 2.181871-0.108619$ \\ $\mathrm{E}=7239.3948011 \mathrm{NIMAG}=0$}

\section{3s-INT at MP2/6-31+G*}

C $\quad 0.050870 \quad 1.795463 \quad-0.013058$

Se $-0.002103-0.176373-0.027463$

Se $2.644981-0.167266-0.027237$

Se $-2.656230 \quad-0.156664 \quad-0.027478$

$\begin{array}{lrll}\mathrm{H} & 2.762159 & -0.098605 & 1.450937\end{array}$

$\mathrm{H} \quad-2.782349-0.087499 \quad 1.450037$

$\begin{array}{llll}\mathrm{H} & 0.416349 & 2.144764 & 0.955656\end{array}$

$\begin{array}{llll}\mathrm{H} & 0.722159 & 2.138302 & -0.801247\end{array}$

$\mathrm{H} \quad-0.969539 \quad 2.140546-0.182978$

$E=-7234.1022946 \mathrm{NIMAG}=0$

\section{3a-INT at B3LYP/6-31+G*}

$\begin{array}{lllll}\text { C } & 0.053138 & 1.841707 & 0.002949\end{array}$

Se $0.002189-0.144046-0.000130$

$\begin{array}{llll}\text { Se } & 2.679631 & -0.189898 & 0.044445\end{array}$

Se $-2.695528-0.177587-0.045132$

$\mathrm{H} \quad 2.849257 \quad-0.101014-1.431815$

$\mathrm{H} \quad-2.868450 \quad-0.125033 \quad 1.432758$

$\begin{array}{llll}\mathrm{H} & 0.573214 & 2.191318 & 0.897533\end{array}$

$\mathrm{H} \quad 0.577313 \quad 2.196172-0.887549$

$\mathrm{H} \quad-0.984092 \quad 2.180371-0.000837$

$\mathrm{E}=-7239.3947206 \mathrm{NIMAG}=0$

\section{3a-INT at MP2/6-31+G*}

$\begin{array}{llll}\text { C } & -0.059181 & 1.796498 & 0.003049\end{array}$

Se $0.002246-0.175367-0.000231$

Se $-2.644391-0.1689760 .044140$

Se $2.657691-0.155544-0.044720$

$\mathrm{H} \quad-2.788073-0.093790 \quad-1.431481$

$\mathrm{H} \quad 2.810837 \quad-0.116345 \quad 1.431355$

$\mathrm{H} \quad-0.591154 \quad 2.145234-0.884166$

$\begin{array}{lrrr}\mathrm{H} & -0.580125 & 2.139618 & 0.898722\end{array}$

$\mathrm{H} \quad 0.975034 \quad 2.142453-0.005159$

$\mathrm{E}=-7234.1021485 \mathrm{NIMAG}=0$

\section{3a-TS at B3LYP/6-31+G* \\ Se $1.187653 \quad 0.939916 \quad-0.002781$ \\ $\begin{array}{llll}\text { C } & -0.411730 & -0.194792 & 0.009394\end{array}$ \\ $\begin{array}{llll}\text { Se } & 2.945985 & -0.637561 & 0.043929\end{array}$ \\ $\mathrm{H} \quad-1.310104 \quad 0.441740 \quad 0.037007$ \\ $\mathrm{H} \quad-0.449048-0.798933-0.897569$ \\ $\mathrm{H} \quad-0.410039-0.832380 \quad 0.893981$ \\ $\mathrm{H} \quad 3.057123-0.876845-1.424994$ \\ Se $-3.967354-0.197325-0.044189$ \\ $\mathrm{H} \quad-4.071216-0.335849 \quad 1.438616$ \\ $\mathrm{E}=-7239.3715967$ NIMAG $=1$}

\section{3a-TS at MP2/6-31+G*}

$\begin{array}{lrrr}\text { Se } & -1.204777 & 0.953470 & -0.000533 \\ \mathrm{C} & 0.358365 & -0.206635 & -0.006587 \\ \mathrm{Se} & -2.904317 & -0.650967 & -0.041229 \\ \mathrm{H} & 1.265714 & 0.406992 & -0.030422 \\ \mathrm{H} & 0.373534 & -0.810951 & 0.900266 \\ \mathrm{H} & 0.337876 & -0.844207 & -0.890367 \\ \mathrm{H} & -2.986790 & -0.888380 & 1.423674 \\ \mathrm{Se} & 3.958173 & -0.190106 & 0.043504 \\ \mathrm{H} & 3.990776 & -0.445143 & -1.422853 \\ \mathrm{E}= & -7234.0806558 & \text { NIMAG }= & 0\end{array}$




4s-TS at B3LYP/6-31+G*
$\begin{array}{lrrr}\text { Se } & 1.310826 & -1.174373 & 0.344521 \\ \mathrm{C} & -0.410231 & -0.553509 & -0.412911 \\ \mathrm{Se} & 2.777374 & 0.468118 & -0.465269 \\ \mathrm{H} & -1.188190 & -1.263001 & -0.117952 \\ \mathrm{H} & -0.672161 & 0.425926 & -0.019915 \\ \mathrm{H} & -0.344474 & -0.522095 & -1.499899 \\ \mathrm{Se} & -3.733757 & 0.201774 & -0.427659 \\ \mathrm{C} & -3.251604 & 0.411866 & 1.492085 \\ \mathrm{H} & -2.878688 & 1.424544 & 1.692688 \\ \mathrm{H} & -4.125468 & 0.237937 & 2.130469 \\ \mathrm{H} & -2.471782 & -0.301907 & 1.792151 \\ \mathrm{C} & 2.418823 & 1.910237 & 0.835117 \\ \mathrm{H} & 3.050573 & 2.760033 & 0.554603 \\ \mathrm{H} & 1.368561 & 2.202994 & 0.783789 \\ \mathrm{H} & 2.668630 & 1.576364 & 1.844152 \\ \mathrm{E}= & -7318.0062066 \text { NIMAG } & =0\end{array}$

\section{4s-TS at MP2/6-31+G*}

$\begin{array}{lrrr}\text { Se } & 1.483009 & -1.233530 & 0.402926 \\ \mathrm{C} & -0.377419 & -0.736830 & 0.051059 \\ \mathrm{Se} & 2.522571 & 0.591007 & -0.597161 \\ \mathrm{C} & 2.239246 & 1.901267 & 0.831671 \\ \mathrm{H} & -1.016109 & -1.523885 & 0.454087 \\ \mathrm{H} & -0.615184 & 0.200676 & 0.547442 \\ \mathrm{H} & -0.548610 & -0.650950 & -1.019011 \\ \mathrm{H} & 2.672937 & 2.847091 & 0.493085 \\ \mathrm{H} & 1.169945 & 2.032141 & 1.004624 \\ \mathrm{H} & 2.731678 & 1.566931 & 1.745959 \\ \mathrm{Se} & -3.646272 & -0.026608 & -0.375640 \\ \mathrm{C} & -3.120299 & 1.174911 & 1.108131 \\ \mathrm{H} & -2.369245 & 1.903723 & 0.778212 \\ \mathrm{H} & -3.986745 & 1.732758 & 1.477773 \\ \mathrm{H} & -2.704327 & 0.605874 & 1.948443 \\ \mathrm{E}= & -7312.421384 & \text { NIMAG } & = \\ \end{array}$

\section{4s-INT at B3LYP/6-31+G*}

$\begin{array}{lrrr}\text { C } & 0.033362 & -0.542193 & 1.793607 \\ \text { Se } & 0.000268 & -0.419228 & -0.193691 \\ \mathrm{Se} & 2.666065 & -0.212094 & -0.180988 \\ \mathrm{Se} & -2.672787 & -0.211326 & -0.197397 \\ \mathrm{H} & -0.990898 & -0.745946 & 2.110988 \\ \mathrm{C} & 2.604272 & 1.750204 & 0.065596 \\ \mathrm{C} & -2.613544 & 1.736960 & 0.149534 \\ \mathrm{H} & 0.384790 & 0.399248 & 2.225290 \\ \mathrm{H} & 0.704939 & -1.350173 & 2.088337 \\ \mathrm{H} & 3.625201 & 2.147961 & 0.050999 \\ \mathrm{H} & -3.631396 & 2.142009 & 0.123083 \\ \mathrm{H} & 2.026312 & 2.210850 & -0.740807 \\ \mathrm{H} & -2.175351 & 1.937899 & 1.132194 \\ \mathrm{H} & 2.138219 & 2.006173 & 1.022535 \\ \mathrm{H} & -2.006943 & 2.232183 & -0.614474 \\ \mathrm{E} & = & -7318.0289525 \text { NIMAG } & = \\ \end{array}$

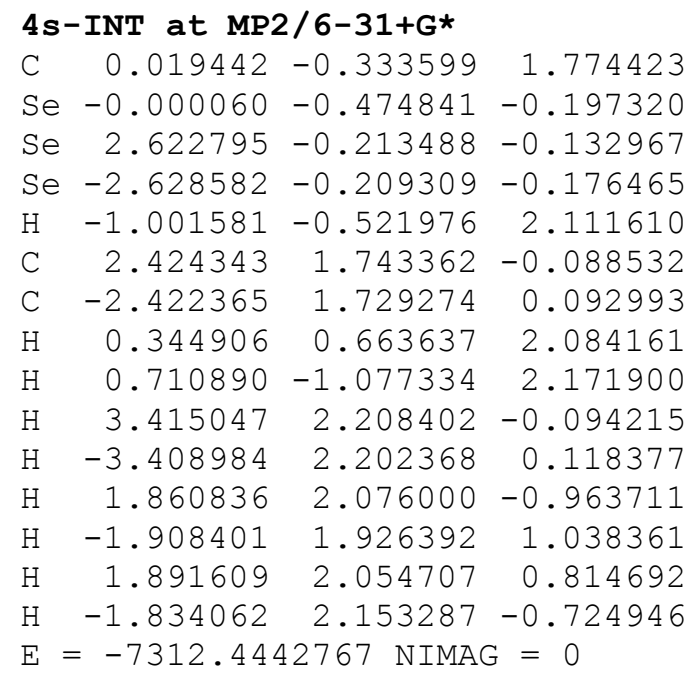

\section{4a-TS at B3LYP/6-31+G*}

$\begin{array}{lrrr}\text { Se } & 1.376588 & 1.270256 & -0.159077 \\ \mathrm{C} & -0.326431 & 0.637212 & -0.948045 \\ \mathrm{Se} & 2.307858 & -0.734373 & 0.601514 \\ \mathrm{C} & 3.192792 & -1.371490 & -1.047062 \\ \mathrm{H} & -0.955876 & 1.524745 & -1.057547 \\ \mathrm{H} & -0.138364 & 0.196754 & -1.928692 \\ \mathrm{H} & -0.855103 & -0.079534 & -0.314968 \\ \mathrm{H} & 3.718358 & -2.297333 & -0.788321 \\ \mathrm{H} & 2.445576 & -1.578745 & -1.815549 \\ \mathrm{H} & 3.907099 & -0.626706 & -1.403996 \\ \mathrm{Se} & -3.587795 & -0.523749 & -0.244343 \\ \mathrm{C} & -3.203805 & 0.682761 & 1.292909 \\ \mathrm{H} & -3.809894 & 1.594883 & 1.232325 \\ \mathrm{H} & -3.426250 & 0.181240 & 2.242029 \\ \mathrm{H} & -2.147004 & 0.981234 & 1.312718 \\ \mathrm{E}= & -7318.0038793 \mathrm{NIMAG} & = & 0\end{array}$

4a-TS at MP2/6-31+G*
$\begin{array}{lrrr}\text { Se } & 1.396858 & 1.276500 & -0.179439 \\ \mathrm{C} & -0.299143 & 0.640925 & -0.931879 \\ \mathrm{Se} & 2.233851 & -0.741213 & 0.611478 \\ \mathrm{C} & 2.892392 & -1.482675 & -1.079679 \\ \mathrm{H} & -0.956539 & 1.509433 & -1.009099 \\ \mathrm{H} & -0.129322 & 0.218420 & -1.922981 \\ \mathrm{H} & -0.781617 & -0.099762 & -0.294253 \\ \mathrm{H} & 3.357062 & -2.446145 & -0.848651 \\ \mathrm{H} & 2.056028 & -1.635836 & -1.763414 \\ \mathrm{H} & 3.627387 & -0.808677 & -1.522422 \\ \mathrm{Se} & -3.512825 & -0.503566 & -0.217659 \\ \mathrm{C} & -3.003819 & 0.723534 & 1.251639 \\ \mathrm{H} & -3.340630 & 1.744342 & 1.038718 \\ \mathrm{H} & -3.459536 & 0.401134 & 2.193014 \\ \mathrm{H} & -1.917450 & 0.747927 & 1.399692 \\ \mathrm{E}= & -7312.4191762 & \text { NIMAG } & =\end{array}$




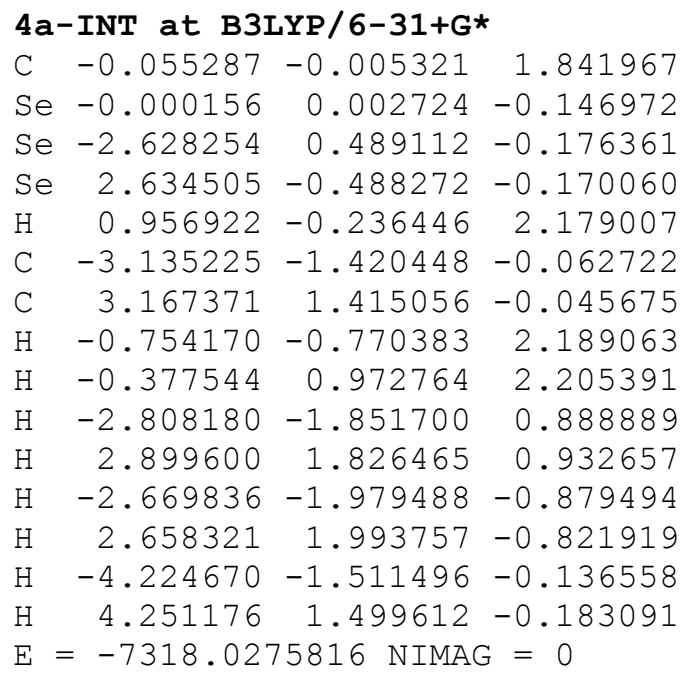

\section{4a-INT at MP2/6-31+G*}

$\begin{array}{lrrr}\text { C } & -0.035429 & 0.164650 & 1.742620 \\ \mathrm{Se} & 0.001473 & -0.026638 & -0.224437 \\ \mathrm{Se} & -2.588731 & 0.461615 & -0.198176 \\ \mathrm{Se} & 2.600666 & -0.485265 & -0.138958 \\ \mathrm{H} & 0.956341 & -0.116236 & 2.102328 \\ \mathrm{C} & -2.995363 & -1.424335 & 0.190610 \\ \mathrm{C} & 2.983107 & 1.438362 & 0.036849 \\ \mathrm{H} & -0.793643 & -0.500990 & 2.160582 \\ \mathrm{H} & -0.275732 & 1.196085 & 2.008458 \\ \mathrm{H} & -2.552778 & -1.721918 & 1.145687 \\ \mathrm{H} & 2.553716 & 1.824010 & 0.965989 \\ \mathrm{H} & -2.586132 & -2.058781 & -0.598999 \\ \mathrm{H} & 2.542668 & 1.977528 & -0.805398 \\ \mathrm{H} & -4.079801 & -1.560903 & 0.244291 \\ \mathrm{H} & 4.065576 & 1.598918 & 0.050000 \\ \mathrm{E} & = & -7312.4421156 \mathrm{NIMAG} & =0\end{array}$

\section{5-TS at B3LYP/aug-cc-pVDZ}

$\begin{array}{lrrr}\mathrm{S} & -2.406163 & 0.488391 & 0.090626 \\ \mathrm{Se} & -0.261983 & -0.433028 & -0.006748 \\ \mathrm{H} & 0.717968 & 0.725693 & -0.010190 \\ \mathrm{~S} & 2.896055 & 0.327167 & -0.077786 \\ \mathrm{H} & -2.609358 & 0.636150 & -1.245874 \\ \mathrm{H} & 2.960536 & 0.312193 & 1.280037 \\ \mathrm{E}= & -3199.8604513 \mathrm{NIMAG}=1\end{array}$

\section{5-TS at MP2/aug-cc-pVDz}

$\begin{array}{lrrr}\mathrm{S} & -2.353255 & 0.515425 & 0.088945 \\ \mathrm{Se} & -0.280989 & -0.449157 & -0.005978 \\ \mathrm{H} & 0.649775 & 0.716139 & -0.012291 \\ \mathrm{~S} & 2.886642 & 0.332926 & -0.077566 \\ \mathrm{H} & -2.543293 & 0.657140 & -1.243483 \\ \mathrm{H} & 2.912953 & 0.324451 & 1.276972 \\ \mathrm{E}= & -3197.122681 \mathrm{NIMAG}=1\end{array}$

\section{5-INT at B3LYP/aug-cC-pVDZ}

$\begin{array}{lrrr}\mathrm{H} & 0.000000 & -0.000045 & 1.383290 \\ \mathrm{Se} & 0.000000 & -0.000045 & -0.090228 \\ \mathrm{~S} & -2.592754 & -0.084214 & 0.051301 \\ \mathrm{~S} & 2.592748 & 0.084307 & 0.051301 \\ \mathrm{H} & -2.769522 & 1.262147 & 0.021402 \\ \mathrm{H} & 2.769611 & -1.262042 & 0.021402 \\ \mathrm{E} & =-3199.8756419 & \mathrm{NIMAG} & =\end{array}$

\section{5-INT at MP2/aug-cc-pVDZ}

$\begin{array}{lrrr}\mathrm{H} & 0.000000 & 0.000000 & -1.353226 \\ \mathrm{Se} & 0.000000 & 0.000000 & 0.108196 \\ \mathrm{~S} & 0.000000 & 2.559828 & -0.072268 \\ \mathrm{~S} & 0.000000 & -2.559828 & -0.072268 \\ \mathrm{H} & -1.347283 & 2.661437 & -0.006424 \\ \mathrm{H} & 1.347283 & -2.661437 & -0.006424 \\ \mathrm{E} & =-3197.1381451 \mathrm{NIMAG}=0\end{array}$

\section{6-TS at B3LYP/aug-cc-pVDZ}

$\begin{array}{lrrr}\text { Se } & -0.241252 & -0.096916 & -0.494128 \\ \mathrm{H} & 0.621949 & 0.332580 & 0.675054 \\ \mathrm{~S} & -2.335711 & -0.666326 & 0.357092 \\ \mathrm{~S} & 2.721737 & 0.816751 & 0.156119 \\ \mathrm{C} & 3.348325 & -0.894335 & 0.443986 \\ \mathrm{H} & 3.827433 & -1.300567 & -0.460960 \\ \mathrm{H} & 4.089888 & -0.909145 & 1.258731 \\ \mathrm{H} & 2.524940 & -1.571631 & 0.721231 \\ \mathrm{C} & -3.176246 & 0.970674 & 0.409329 \\ \mathrm{H} & -2.656010 & 1.651073 & 1.095389 \\ \mathrm{H} & -3.211599 & 1.420773 & -0.590975 \\ \mathrm{H} & -4.202939 & 0.807212 & 0.770626 \\ \mathrm{E} & =-3278.4755581 \mathrm{NIMAG} & = & 1\end{array}$

$\begin{array}{lrrr}\text { 6-TS at MP2/aug-cC-pVDZ } \\ \text { Se }-0.226685 & -0.037489 & -0.520716 \\ \text { H } & 0.593400 & 0.326498 & 0.676479 \\ \text { S } & -2.247152 & -0.694741 & 0.321597 \\ \text { S } & 2.698806 & 0.794364 & 0.196582 \\ \mathrm{C} & 3.077868 & -0.989423 & 0.444608 \\ \mathrm{H} & 3.597337 & -1.412942 & -0.433198 \\ \mathrm{H} & 3.721620 & -1.142304 & 1.328750 \\ \mathrm{H} & 2.147509 & -1.568940 & 0.593610 \\ \mathrm{C} & -3.059390 & 0.938524 & 0.496176 \\ \mathrm{H} & -2.507772 & 1.562196 & 1.215204 \\ \mathrm{H} & -3.096385 & 1.449751 & -0.477324 \\ \mathrm{H} & -4.085742 & 0.771795 & 0.865263 \\ \mathrm{E} & -1.3275 .4807744 \mathrm{NIMAG} & = & 1\end{array}$




\section{6-INT at B3LYP/aug-cc-pVDZ}

Se $0.000017-0.000390-0.118096$

$\mathrm{H} \quad 0.000017-0.000390 \quad 1.356650$

S $\quad-2.463198-0.754558 \quad 0.019125$

S $\quad 2.462872 \quad 0.754953 \quad 0.019125$

$\begin{array}{lrrr}\text { C } & -3.279672 & 0.894224 & 0.108299\end{array}$

C $\quad 3.280132-0.893440 \quad 0.108299$

$\mathrm{H} \quad 3.075370-1.390296 \quad 1.068208$

$\mathrm{H} \quad-3.074673 \quad 1.390982 \quad 1.068208$

H $\quad 2.929265-1.547728-0.702556$

$\mathrm{H} \quad-2.928493 \quad 1.548345 \quad-0.702556$

$\mathrm{H} \quad 4.368933-0.764671 \quad 0.007858$

$\mathrm{H}-4.368535 \quad 0.765974 \quad 0.007858$

$\mathrm{E}=-3278.4892898 \mathrm{NIMAG}=0$

\section{6-INT at MP2/aug-cc-pVDZ}

$\begin{array}{lrrr}\text { Se } & 0.000001 & -0.000022 & -0.137427 \\ \mathrm{H} & 0.000001 & -0.000022 & 1.326243 \\ \mathrm{~S} & -2.418028 & -0.761957 & 0.045704 \\ \mathrm{~S} & 2.418008 & 0.761980 & 0.045704 \\ \mathrm{C} & -3.128218 & 0.930160 & 0.101225 \\ \mathrm{C} & 3.128246 & -0.930116 & 0.101225 \\ \mathrm{H} & 2.815127 & -1.456455 & 1.018100 \\ \mathrm{H} & -2.815084 & 1.456490 & 1.018100 \\ \mathrm{H} & 2.788942 & -1.516624 & -0.768151 \\ \mathrm{H} & -2.788897 & 1.516658 & -0.768151 \\ \mathrm{H} & 4.230278 & -0.872536 & 0.084566 \\ \mathrm{H} & -4.230251 & 0.872611 & 0.084566\end{array}$

\section{7-TS at B3LYP/aug-CC-pVDZ}

$\begin{array}{lrrr}\mathrm{S} & -0.605544 & -0.281179 & 0.533871 \\ \mathrm{H} & -1.267152 & 0.028034 & -0.621990 \\ \mathrm{Se} & 1.596436 & -0.396792 & -0.153885 \\ \mathrm{~S} & -3.665305 & 0.274124 & -0.172588 \\ \mathrm{H} & -3.893306 & -1.044785 & -0.414001 \\ \mathrm{C} & 2.101470 & 1.510624 & 0.042872 \\ \mathrm{H} & 3.172225 & 1.598196 & -0.189245 \\ \mathrm{H} & 1.518248 & 2.123561 & -0.653124 \\ \mathrm{H} & 1.915910 & 1.835064 & 1.072689 \\ \mathrm{E}= & -3239.1713473 \mathrm{NIMAG}=1\end{array}$

\section{7-TS at MP2/aug-cC-pVDZ}

$\begin{array}{lrrr}\text { Se } & -0.611897 & -0.485797 & -0.012904 \\ \text { C } & 0.351884 & 1.207578 & 0.016034 \\ \text { S } & -2.716629 & 0.282266 & 0.092209 \\ \text { S } & 3.735568 & 0.000156 & -0.076395 \\ \text { H } & -2.910699 & 0.470140 & -1.235183 \\ \text { H } & 3.683293 & -0.184362 & 1.265938 \\ \text { H } & 0.091529 & 1.792255 & -0.877332 \\ \mathrm{H} & 0.098323 & 1.754259 & 0.934656 \\ \mathrm{H} & 1.427715 & 0.920582 & 0.001426 \\ \mathrm{E}= & -3236.3060679 \text { NIMAG }=1\end{array}$

\section{7-INT at B3LYP/aug-CC-PVDZ}

$\begin{array}{lrrr}\text { S } & 0.878481 & -0.135212 & -0.125954 \\ \mathrm{H} & 0.942125 & -0.128556 & 1.221847 \\ \mathrm{Se} & -1.621121 & -0.434353 & 0.014511 \\ \mathrm{~S} & 3.475708 & 0.230286 & 0.028130 \\ \mathrm{H} & 3.757369 & -1.094196 & -0.077936 \\ \mathrm{C} & -2.107025 & 1.489427 & -0.005678 \\ \mathrm{H} & -3.197759 & 1.577343 & -0.106360 \\ \mathrm{H} & -1.787288 & 1.974108 & 0.925075 \\ \mathrm{H} & -1.621210 & 1.981550 & -0.856767 \\ \mathrm{E} & =-3239.1761422 & \text { NIMAG }=0\end{array}$

\section{7-INT at MP2/aug-cC-pVDZ}

$\begin{array}{lrrr}\mathrm{S} & 0.861792 & -0.163463 & -0.145630 \\ \mathrm{H} & 0.909723 & -0.180501 & 1.198678 \\ \mathrm{Se} & -1.620723 & -0.422282 & 0.019887 \\ \mathrm{~S} & 3.398516 & 0.218615 & 0.040022 \\ \mathrm{H} & 3.667974 & -1.100721 & -0.092422 \\ \mathrm{C} & -1.940123 & 1.518781 & -0.007618 \\ \mathrm{H} & -3.023024 & 1.710743 & -0.079408 \\ \mathrm{H} & -1.545411 & 1.978098 & 0.910844 \\ \mathrm{H} & -1.428866 & 1.954860 & -0.878415 \\ \mathrm{E} & =-3236.3099565 & \text { NIMAG }=0\end{array}$

\section{8-TSn at B3LYP/aug-CC-PVDZ}

$\begin{array}{lrrr}\mathrm{S} & 0.346914 & 0.615870 & 0.010440 \\ \mathrm{H} & 1.104085 & -0.532236 & 0.043470 \\ \mathrm{Se} & -1.846685 & -0.155080 & -0.047336 \\ \mathrm{~S} & 3.420233 & -0.224287 & 0.078660 \\ \mathrm{H} & -2.066073 & -0.235640 & 1.420389 \\ \mathrm{H} & 3.474932 & -0.224726 & -1.280031 \\ \mathrm{E}= & -3199.8575146 \mathrm{~N} & \text { IMAG }=1\end{array}$

\section{8-TSn at MP2/aug-cc-pVDz}

$\begin{array}{lrrr}\mathrm{S} & 0.341606 & 0.566433 & 0.007731 \\ \mathrm{H} & 0.998452 & -0.622441 & 0.032862 \\ \mathrm{Se} & -1.827953 & -0.141164 & -0.046081 \\ \mathrm{~S} & 3.392228 & -0.201224 & 0.079673 \\ \mathrm{H} & -2.036714 & -0.205344 & 1.409586 \\ \mathrm{H} & 3.447343 & -0.215972 & -1.274149 \\ \mathrm{E} & = & -3197.1192287 & \mathrm{NIMAG}= \\ \end{array}$

\section{8-INT at B3LYP/aug-cC-pVDZ}

S $\quad 0.740492 \quad 0.021128 \quad-0.124555$

H $\quad 0.709237 \quad 0.016248 \quad 1.224139$

Se $-1.919214-0.049339 \quad 0.011231$

$\begin{array}{llll}\text { S } & 3.213359 & 0.073406 & 0.027164\end{array}$

$\mathrm{H} \quad-2.082553 \quad 1.423751-0.019154$

H $\quad 3.364963-1.275024-0.028566$

$\mathrm{E}=-3199.8670713 \mathrm{NIMAG}=0$ 
$E=-3275.4722001 \mathrm{NIMAG}=1$

\section{8-INT at MP2/aug-cc-pVDZ}

$\begin{array}{lrrr}\mathrm{S} & 0.715251 & 0.018215 & -0.143647 \\ \mathrm{H} & 0.694656 & 0.021000 & 1.200947 \\ \mathrm{Se} & -1.888182 & -0.047975 & 0.016774 \\ \mathrm{~S} & 3.174072 & 0.073443 & 0.038452 \\ \mathrm{H} & -2.014195 & 1.415324 & -0.042149 \\ \mathrm{H} & 3.288572 & -1.271695 & -0.045982 \\ \mathrm{E}= & -3197.1279305 & \mathrm{NIMAG}=0\end{array}$

\section{8-TSx at B3LYP/aug-cC-pVDZ}

$\begin{array}{lrrr}\mathrm{S} & 1.192784 & 0.574446 & -0.038322 \\ \mathrm{H} & 0.360862 & -0.512534 & 0.081809 \\ \mathrm{~S} & 3.185963 & -0.305728 & 0.101946 \\ \mathrm{Se} & -2.105771 & -0.095704 & -0.038726 \\ \mathrm{H} & 3.374641 & -0.577290 & -1.218284 \\ \mathrm{H} & -2.199217 & 0.044261 & 1.435192 \\ \mathrm{E} & =-3199.8597687 & & -0.04 M A G \\ & = & \end{array}$

\section{8-TSx at MP2/aug-cC-pVDZ}

$\begin{array}{lrrr}\mathrm{S} & 1.168719 & 0.512350 & -0.040915 \\ \mathrm{H} & 0.449791 & -0.628314 & 0.106760 \\ \mathrm{~S} & 3.168784 & -0.264041 & 0.101932 \\ \mathrm{Se} & -2.089470 & -0.084398 & -0.038147 \\ \mathrm{H} & 3.360140 & -0.531248 & -1.211460 \\ \mathrm{H} & -2.168009 & 0.056166 & 1.425429 \\ \mathrm{E}= & -3197.1218374 \mathrm{NIMAG}=1\end{array}$

\section{9-TSn at B3LYP/aug-cC-pVDZ}

$\begin{array}{lrrr}\text { S } & 0.385249 & -0.073293 & -0.524301 \\ \mathrm{Se} & 2.580690 & 0.148308 & 0.006102 \\ \mathrm{~S} & -3.649202 & -0.592846 & -0.253230 \\ \mathrm{H} & 2.977214 & -1.276615 & -0.163985 \\ \mathrm{C} & -3.810326 & 1.195581 & 0.185092 \\ \mathrm{H} & -4.068235 & 1.804286 & -0.697526 \\ \mathrm{H} & -4.598747 & 1.356977 & 0.939720 \\ \mathrm{H} & -2.869745 & 1.602547 & 0.597624 \\ \mathrm{C} & -0.395910 & -0.529987 & 1.066845 \\ \mathrm{H} & -0.003961 & -1.488823 & 1.426224 \\ \mathrm{H} & -0.243373 & 0.257737 & 1.813720 \\ \mathrm{H} & -1.475970 & -0.633898 & 0.805632 \\ \mathrm{E} & =-3278.4653828 & -1.01 M A G & =\end{array}$

\section{9-TSn at MP2/aug-cc-pVDZ}

$\begin{array}{lrrr}\text { S } & 0.610559 & -0.753013 & -0.309795 \\ \mathrm{Se} & 2.607183 & 0.193585 & -0.020349 \\ \mathrm{C} & -0.499063 & 0.430278 & 0.521869 \\ \mathrm{~S} & -3.789140 & -0.633257 & 0.149530 \\ \mathrm{H} & 2.888685 & -0.365544 & 1.314124 \\ \mathrm{C} & -3.933304 & 1.143117 & -0.323379 \\ \mathrm{H} & -2.957983 & 1.666620 & -0.258299 \\ \mathrm{H} & -4.295217 & 1.260461 & -1.361408 \\ \mathrm{H} & -4.635740 & 1.684751 & 0.336552 \\ \mathrm{H} & -0.242432 & 0.498365 & 1.588797 \\ \mathrm{H} & -0.425337 & 1.414797 & 0.039435 \\ \mathrm{H} & -1.524707 & -0.001372 & 0.405958\end{array}$

\section{9-INT at B3LYP/aug-cC-pVDZ}

S $\quad 0.569052-0.053081-0.334383$

$\begin{array}{llll}\text { C } & 0.510500 & 1.658470 & 0.356221\end{array}$

S $\quad 2.773853-0.124350-0.647816$

Se $-2.522064-0.219096 \quad 0.092496$

C $\quad 3.354395-0.623413 \quad 1.024772$

H $2.839299-1.536792 \quad 1.347654$

$\begin{array}{lllll}\mathrm{H} & 4.435503 & -0.817261 & 0.963937\end{array}$

$\mathrm{H} \quad 3.178311 \quad 0.173535 \quad 1.759891$

$\mathrm{H} \quad 1.093316 \quad 1.715739 \quad 1.285174$

$\mathrm{H} \quad 0.906146 \quad 2.371529-0.377413$

$\mathrm{H} \quad-0.546643 \quad 1.856220 \quad 0.562383$

$\mathrm{H} \quad-2.831600 \quad 0.314839-1.257269$

$E=-3278.4835988 \mathrm{NIMAG}=0$

\section{9-INT at MP2/aug-cc-pVDZ}

S $\quad 0.671809-0.117979-0.387161$

$\begin{array}{llll}\text { C } & 0.508427 & 1.545950 & 0.366146\end{array}$

S $\quad 2.801608-0.086635-0.627981$

Se $-2.567026-0.1966390 .091096$

C $\quad 3.312504-0.568507 \quad 1.062438$

H $\quad 2.860734-1.535626 \quad 1.326457$

$\mathrm{H} \quad 4.412015-0.653974 \quad 1.075038$

$\mathrm{H} \quad 3.003177 \quad 0.195707 \quad 1.791858$

H $\quad 1.074520 \quad 1.593381 \quad 1.309858$

$\mathrm{H} \quad 0.874277 \quad 2.309462 \quad-0.336138$

$\mathrm{H} \quad-0.569522 \quad 1.655651 \quad 0.558737$

$\mathrm{H} \quad-2.876588 \quad 0.530302-1.152305$

$\mathrm{E}=-3275.4908921 \mathrm{NIMAG}=0$

\section{9-TSX at B3LYP/aug-cC-pVDZ}

S $\quad-0.998377-0.229011-0.450776$

S $\quad-3.105097-0.447131-0.344618$

Se $2.930451 \quad 0.181456-0.061162$

C $\quad-0.488016-0.629195 \quad 1.267551$

C $\quad-3.640983 \quad 1.197020 \quad 0.286624$

$\mathrm{H} \quad 3.181461-1.218890-0.487400$

$\mathrm{H} \quad-0.920440 \quad 0.089148 \quad 1.975455$

$\mathrm{H} \quad-0.784447-1.653664 \quad 1.522579$

$\mathrm{H} \quad 0.610451-0.525543 \quad 1.242186$

$\mathrm{H} \quad-3.287328 \quad 1.992494-0.379792$

$\begin{array}{llll}\mathrm{H} & -4.740690 & 1.191501 & 0.304564\end{array}$

$\mathrm{H} \quad-3.264768 \quad 1.366773 \quad 1.303174$

$E=-3278.4824883 \mathrm{NIMAG}=1$ 
9-TSx at MP2/aug-cc-pVDZ

S $\quad-1.009207-0.459730-0.341388$

$\begin{array}{lrrr}\mathrm{C} & -0.464891 & 0.087604 & 1.313274\end{array}$

$\mathrm{S} \quad-3.088162-0.547233-0.051388$

$\begin{array}{llll}\text { Se } & 2.890861 & 0.125865 & -0.123714\end{array}$

C $\quad-3.524004 \quad 1.216894 \quad-0.268629$

$\mathrm{H} \quad-3.213256 \quad 1.558956-1.265990$

$\mathrm{H} \quad-4.618484 \quad 1.304045-0.165657$

$\begin{array}{llll}\mathrm{H} & -3.037672 & 1.828785 & 0.505537\end{array}$

$\mathrm{H} \quad-0.904116 \quad 1.067843 \quad 1.554442$

$\mathrm{H} \quad-0.745282-0.661701 \quad 2.067539$

$\begin{array}{llll}\mathrm{H} & 0.634891 & 0.174244 & 1.212437\end{array}$

$\mathrm{H} \quad 3.085911-1.267170 \quad 0.314505$

$\mathrm{E}=-3275.4904843 \mathrm{NIMAG}=1$

\section{0-TSn at B3LYP/aug-cC-pVDZ}

$\begin{array}{llll}\mathrm{S} & -0.605544 & -0.281179 & 0.533871\end{array}$

$\mathrm{H} \quad-1.267152 \quad 0.028034 \quad-0.621990$

Se $1.596436-0.396792-0.153885$

S $\quad-3.665305 \quad 0.274124 \quad-0.172588$

$\mathrm{H} \quad-3.893306-1.044785-0.414001$

$\begin{array}{llll}\text { C } & 2.101470 & 1.510624 & 0.042872\end{array}$

$\mathrm{H} \quad 3.172225 \quad 1.598196-0.189245$

$\mathrm{H} \quad 1.518248 \quad 2.123561 \quad-0.653124$

$\mathrm{H} \quad 1.915910 \quad 1.835064 \quad 1.072689$

$\mathrm{E}=-3239.1713473 \mathrm{NIMAG}=1$

\section{0-TSn at MP2/aug-cc-pVDZ}

$\mathrm{S} \quad-0.592217-0.283184 \quad 0.482319$

$\mathrm{H} \quad-1.157348-0.003937-0.715809$

Se $1.590141-0.391677-0.136221$

S $\quad-3.616831 \quad 0.262292-0.150993$

$\mathrm{H} \quad-3.873507-1.046666-0.390095$

C $\quad 2.007240 \quad 1.517898 \quad 0.037888$

$\mathrm{H} \quad 3.082839 \quad 1.654994 \quad-0.161086$

$\mathrm{H} \quad 1.415526 \quad 2.091836-0.688647$

$\mathrm{H} \quad 1.769043 \quad 1.847685 \quad 1.058589$

$E=-3236.3060672$ NIMAG $=1$

\section{0-INT at B3LYP/aug-cC-pVDZ}

S $\quad 0.878481-0.135212-0.125954$

H $\quad 0.942125-0.128556 \quad 1.221847$

Se $-1.621121-0.434353 \quad 0.014511$

$\begin{array}{llll}\text { S } & 3.475708 & 0.230286 & 0.028130\end{array}$

H $\quad 3.757369-1.094196-0.077936$

C $\quad-2.107025 \quad 1.489427-0.005678$

$\mathrm{H} \quad-3.197759 \quad 1.577343-0.106360$

$\mathrm{H} \quad-1.787288 \quad 1.974108 \quad 0.925075$

$\mathrm{H} \quad-1.621210 \quad 1.981550 \quad-0.856767$

$\mathrm{E}=-3239.1761422 \mathrm{NIMAG}=0$

\section{0-INT at MP2/aug-cc-pVDZ}

S $\quad 0.861792-0.163463-0.145630$

H $\quad 0.909723-0.180501 \quad 1.198678$

Se $-1.620723-0.422282 \quad 0.019887$

S $\quad 3.398516 \quad 0.2186150 .040022$

H $\quad 3.667974-1.100721-0.092422$

C $\quad-1.940123 \quad 1.518781-0.007618$

$\mathrm{H} \quad-3.023024 \quad 1.710743-0.079408$

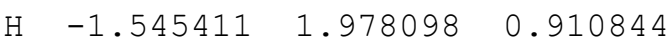

$\mathrm{H} \quad-1.428866 \quad 1.954860-0.878415$

$E=-3236.3099565 \mathrm{NIMAG}=0$

\section{0-TSX at B3LYP/aug-cC-pVDZ}

S $-1.400092-0.171954 \quad 0.570908$

$\mathrm{H} \quad-0.532873-0.189313-0.505889$

S $\quad-3.377590 \quad 0.234937-0.301506$

Se $\quad 1.813517-0.432938-0.105806$

$\mathrm{H} \quad-3.751014-1.050596-0.545674$

$\begin{array}{llll}\text { C } & 2.101051 & 1.536218 & 0.029047\end{array}$

H $\quad 2.722120 \quad 1.889125 \quad-0.806332$

$\mathrm{H} \quad 1.135183 \quad 2.059100 \quad-0.003141$

$\mathrm{H} \quad 2.603628 \quad 1.786533 \quad 0.973715$

$E=-3239.164162 \mathrm{NIMAG}=1$

\section{0-TSx at MP2/aug-cc-pVDZ}

S $\quad-1.323937-0.264321 \quad 0.509334$

$\mathrm{H} \quad-0.559359-0.219078-0.619582$

S $\quad-3.284881 \quad 0.223530-0.258534$

Se $\quad 1.831724-0.395268-0.101999$

$\mathrm{H} \quad-3.683780-1.033161-0.566721$

C $\quad \begin{array}{llll}1.751425 & 1.577470 & 0.060104\end{array}$

H $\quad 2.327463 \quad 2.058090 \quad-0.747542$

$\mathrm{H} \quad 0.703926 \quad 1.918802 \quad-0.000252$

$\mathrm{H} \quad 2.165675 \quad 1.902272 \quad 1.028650$

$\mathrm{E}=-3236.2976328 \mathrm{NIMAG}=1$ 